\title{
IMAge DEBlocking IN WAVELET DOMAIN BASED ON Local Laplace Prior
}

\author{
Vijay Kumar Nath and Deepika Hazarika \\ Department of Electronics and Communication Engineering, School of Engineering, \\ Tezpur University (A Central University), Napaam, Tezpur, Assam, India. \\ vknath@tezu.ernet.in and deepika@tezu.ernet.in
}

\begin{abstract}
This paper presents a efficient non iterative wavelet based image deblocking method which employs a maximum a posteriori (MAP) estimator that uses the Laplace probability density function with local variance to smooth out the blocking artifacts in block discrete cosine transform (DCT) compressed images. We have shown that the noise standard deviation has a strong relationship with the average of first $3 \times 3$ values from the quantization table. The method is adaptive to different block DCT compressed images that are compressed at various bit rates. The proposed method outperforms several well known wavelet and non wavelet based methods both in terms of visual quality and peak signal to noise ratio (PSNR).
\end{abstract}

\section{KEYWORDS}

Block DCT, Wavelet transform, Laplace probability density function, Quantization, Blocking artifacts, MAP estimator, Image deblocking.

\section{INTRODUCTION}

The block DCT [1] is a very important component in several image and video compression schemes such as JPEG, MPEG1, MPEG2 and H.261 [2]. At high compression ratios, the block DCT based methods often exhibits blocking artifacts. Blocking artifacts are the artificial block boundaries which are visually annoying particularly in still images. The main challenge in the image deblocking methods is to effectively smooth out the blocking artifacts while preserving the true edges and other important details.

Many wavelet and non wavelet based methods have been proposed to reduce the blocking artifacts in block DCT compressed images. Reeve and Lim [3] reduced the blocking artifacts using a $3 \times 3$ Gaussian filter at the block boundaries. The main problem with this simple method is that the true edges at the block boundaries may also get blurred. Minami and Zakhor [4] proposed to reduce the blocking artifacts by minimizing a criterion called mean squared difference of slope (MSDS). The projection onto convex sets (POCS) algorithms $[5,6,7,8]$ have shown good results but the main problem with the POCS method is its iterative nature and the high computational complexity. Xu et al. [9] proposed to reduce the blocking artifacts in discrete hadamard transform (DHT) domain. They calculate the block activities in the DHT domain to classify the smooth and coarse regions and according to the block activities the blocking artifacts were adaptively filtered. $\mathrm{Wu}$ et al. [10] proposed a wavelet based postprocessing technique to remove the blocking artifacts based on soft thresholding. The techniques discussed in [11,12] use an overcomplete wavelet representation to reduce the blocking artifacts. Averbuch et al. [13] proposed to apply weighted sums on pixel quartets which are symmetrically aligned to the block boundaries to suppress the blocking artifacts. The image deblocking scheme based on pointwise shape adaptive DCT (SA-DCT) [14] have shown excellent state of the art results which is able to remove both

DOI : 10.5121/ijma.2012.4104 
The International Journal of Multimedia \& Its Applications (IJMA) Vol.4, No.1, February 2012

the blocking and ringing artifacts. Zhai et al. [15] proposed an image deblocking scheme through post filtering in shifted windows of image blocks for JPEG compressed images. Zhang et al. [16] proposed an image deblocking scheme based on adaptive bilateral filter where texture regions and block boundary discontinuities are first detected and based on that the bilateral filter parameters are selected. Nath et al. [17] proposed an image deblocking technique based on adaptive bilateral filter where the bilateral filter parameters were selected based on empirical study. The bilateral filter parameters were made adaptive to the quantization tables.

In this paper, we propose a non iterative, spatially adaptive, wavelet based image deblocking method which is based on modelling the wavelet coefficients in each subbands using a Laplace pdf with local variance. The noise standard deviation is made adaptive to the quantization tables used in the compression process.

This paper is organized as follows. The proposed wavelet domain image deblocking technique is described in Section 2. The experimental results are presented in Section 3. Finally, the conclusions are given in Section 4.

\section{Proposed Wavelet Domain Image Deblocking Method}

We model the degradation due to the quantization noise as some additive noise. We use the following observation model

$$
d=z+n
$$

where $z$ is the original uncompressed image, $d$ is the observed block DCT compressed image and $n$ is the degradation due to quantization process. The wavelet coefficients of the image $d$ is given by

$$
D(k)=Z(k)+N(k)
$$

where $Z(k)$ represents the wavelet coefficients of the uncompressed image. We use a MAP estimator to estimate $Z(k)$ from the noisy observation $D(k)$ which is given by $[18,19]$

$$
Z(k)=\underset{Z(k)}{\arg \max } P_{Z(k) \mid D(k)}(Z(k) \mid D(k))
$$

The above equation can also be written as

$$
Z(k)=\underset{Z(k)}{\arg \max }\left[P_{N}(D(k)-Z(k)) P_{Z(k)}(Z(k))\right]
$$

We assume the noise to be zero mean Gaussian with variance $\sigma_{N}^{2}$ for the similar reasons described in [20]

$$
P_{N}(N(k))=\frac{1}{\sqrt{2 \pi} \sigma_{N}} \exp \left(-\frac{N^{2}(k)}{2 \sigma_{N}^{2}}\right)
$$

Substituting Equation (5) in (4), we have

$$
Z(k)=\underset{Z(k)}{\arg \max }\left[-\frac{(D(k)-Z(k))^{2}}{2 \sigma_{N}^{2}}+f(Z(k))\right]
$$

where $f(Z(k))=\log \left(P_{Z(k)}(Z(k))\right)$. Hence, the MAP estimate of $Z(k)$ is computed by setting the derivative with respect to $Z(k)$ equals to zero

$$
\frac{D(k)-Z(k)}{\sigma_{N}^{2}}+f^{\prime}(Z(k))=0
$$

We model the wavelet coefficients using Laplace pdf with local variance $[18,19]$, hence

$$
P_{Z(k)}(Z(k))=\frac{1}{\sigma_{Z}(k) \sqrt{2}} \exp \left(-\frac{\sqrt{2}|Z(k)|}{\sigma_{Z}(k)}\right)
$$


The International Journal of Multimedia \& Its Applications (IJMA) Vol.4, No.1, February 2012

Solving the above equations, we can write as $[18,19]$

$$
Z(k)=\operatorname{sign}(D(k))\left(|D(k)|-\frac{\sqrt{2} \sigma_{N}^{2}}{\sigma_{Z}(k)}\right)_{+}
$$

From the above, $(a)_{+}$can be defined as

$$
(a)_{+}=\left\{\begin{array}{l}
0, a<0 \\
a, \text { otherwise }
\end{array}\right.
$$

If the SOFTWA operator [18] is defined as

$$
\operatorname{SOFTWA}(p, \eta)=\operatorname{sign}(p)(|p|-\eta)_{+}
$$

Equation (9) can be written as

$$
Z(k)=\operatorname{SOFTWA}\left(D(k), \frac{\sqrt{2} \sigma_{N}^{2}}{\sigma_{Z}(k)}\right)
$$

For each noisy wavelet coefficient, an estimate of $\sigma_{Z}^{2}(k)$ is formed based on its local neighbourhood $B(k)$. Here, we use a square window $B(k)$ centred at $D(k)$. The estimate for $\sigma_{Z}^{2}(k)$ is given by $[18,19]$

$$
\sigma_{Z}^{2}(k)=\max \left(0, \frac{1}{M} \sum_{j \in B(k)} D^{2}(j)-\sigma_{N}^{2}\right)
$$

where $M$ is the number of coefficients in $B(k)$.

To use the Equation (12) to find an estimate of $Z(k)$, we need to find a suitable value of $\sigma_{N}^{2}$. It is to be noted that the $\sigma_{N}$ is not an estimate of the standard deviation of the difference between the original and the block DCT compressed image - $\sigma_{N}$ is just the assumed value of standard deviation of $n$ in our observation model (1). It is assumed that $\sigma_{N}$ is the standard deviation of the noise when added to the original image $z$ would require, for removal, the same amount of adaptive smoothing necessary to reduce the blocking artifacts produced by the block DCT based compression scheme with the quantization table Q. Much larger values of $\sigma_{N}$ may result in oversmoothing and much smaller values may result in insufficient removal of blocking artifacts.

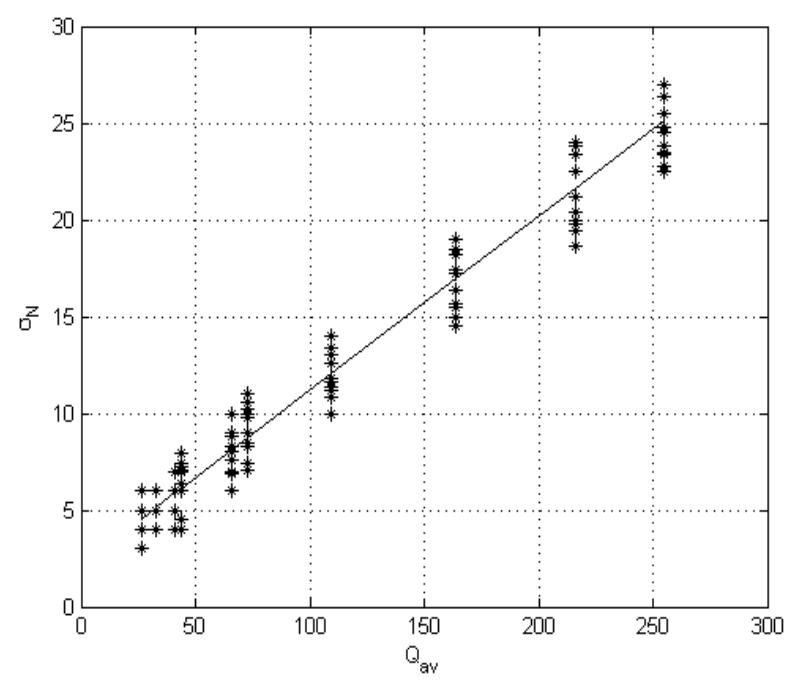

Figure 1. Scatter plot of $\sigma_{N}$ versus $Q_{a v(3 x 3)}$ and curve fitting using linear polynomial 
Motivated by the strategy as discussed in [14], we performed experiments on a large number of test images in which we first plot the best values of $\sigma_{N}$ (found experimentally) which gives lowest mean square error for different compression ratios (different compression ratios means different values of $\left.Q_{a v(3 \times 3)}\right)$.

$$
Q_{a v(3 x 3)}=\frac{1}{9} \sum_{\mathrm{i}, \mathrm{j}=1}^{3} Q_{i, j}
$$

$Q_{a v(3 x 3)}$ is the average of first $3 \times 3$ values from the quantization matrix $\left(Q_{i, j}\right)$.

The data when fitted with a linear polynomial, gives $\sigma_{N}$ as

$$
\sigma_{N}=a_{1} \cdot Q_{a v}+a_{2}
$$

where $a_{1}=0.09$ and $a_{2}=2.2$.

The experimental results show that the results are not very sensitive to the fitting errors and the fitted curve can be considered for a wide range of test images and quantization tables. The $\sigma_{N}$ values are larger for higher compression ratios and smaller for lower compression ratios. Finally the DCT quantization constraint and the range constraint is imposed onto the filtered output (output image after the inverse 2D DWT). The experimental results to be presented in next section will show that this technique reduce the blocking artifacts very efficiently while preserving edges and textures.

\subsection{Quantization Constraint and Range Constraint}

In the POCS based deblocking schemes, the final deblocked image is constrained with the quantization constraint set. The quantization constraint found from the quantization table restricts the value of the DCT coefficients to be within the quantization interval. The deblocked image should satisfy the quantization constraint, to be consistent with the original image. The output image after the inverse 2D DWT (say $\tilde{b}$ ) generally do not satisfy the quantization constraint, so it is projected onto the quantization constraint set to obtain a deblocked image which is consistent with the original image.

Let $m$ represents the set of DCT coefficients of image $\tilde{b}$. The $(\mathrm{u}, \mathrm{v})^{\text {th }}$ DCT coefficient at block position $(\mathrm{x}, \mathrm{y})$ is modified as follows

$$
m_{x y}(u, v)=\left\{\begin{array}{l}
C_{\max }(u, v), \text { if } m_{x, y}(u, v)>C_{\max }(u, v) \\
C_{\min }(u, v), \text { if } m_{x, y}(u, v)<C_{\min }(u, v) \\
m_{x, y}(u, v), \text { otherwise }
\end{array}\right.
$$

where

$$
\begin{gathered}
C_{\max }(u, v)=\left(q_{x, y}(u, v)+0.5\right) \Delta(u, v) \\
C_{\min }(u, v)=\left(q_{x, y}(u, v)-0.5\right) \Delta(u, v)
\end{gathered}
$$

For $u=0, \ldots \ldots \ldots . .7, v=0, \ldots \ldots \ldots .7, x=0, \ldots \ldots($ row $/ 8)-1, y=0, \ldots \ldots \ldots .(\mathrm{col} / 8)-1$. The quantized coefficients $(q)$ that is available at the decoder input is given by

$$
q_{x, y}(u, v)=\text { round }\left[\frac{m_{a, b}(u, v)}{\Delta(u, v)}\right]
$$

where $m$ is the original image DCT coefficients and $\Delta(u, v)$ represents the quantization interval of the $(\mathrm{u}, \mathrm{v})^{\text {th }}$ DCT coefficient. A narrow quantization constraint set discussed in [21], produces 
The International Journal of Multimedia \& Its Applications (IJMA) Vol.4, No.1, February 2012

better results in terms of mean square error. For narrow quantization constraint set, the $C_{\max }(u, v)$ and $C_{\min }(u, v)$ is given by

$$
\begin{gathered}
C_{\max }(u, v)=\left(q_{x, y}(u, v)+\varsigma_{1}\right) \Delta(u, v) \\
C_{\min }(u, v)=\left(q_{x, y}(u, v)-\varsigma_{2}\right) \Delta(u, v)
\end{gathered}
$$

where $0<\varsigma_{1}, \varsigma_{2} \leq 0.5$. We use $\varsigma_{1}=0.3$ and $\varsigma_{2}=0.3$ for the experiments. The inverse block DCT with the modified coefficients is performed to obtain the deblocked image. Finally the range constraint is applied on this image. Since the 8 bit original images used in the experiments have values in the range of (0-255), the deblocked image must also satisfy this range. Any value outside the range of $(0-255)$ is projected into this given range.

\section{EXPERIMENTAL RESULTS}

The proposed method has been tested on a large number of various test images compressed by the JPEG standard and the quantization tables Q1, Q2 and Q3 [10,12] which are commonly used in literature for simulating various types of block DCT compression. In this paper, we report our results only for Lena, Barbara and Peppers image. The baseline IJG JPEG implementation [22] is used for the simulation of all JPEG experiments presented in this paper. We used a wavelet transform with Daubechies-8 wavelet and four levels of decomposition. Center square shaped window of size $5 \times 5$ is used in the wavelet domain to estimate the variances.

Table 1. PSNR comparison between the proposed method and best results reported in [13] for restoration from JPEG compression with different Quality factors.

\begin{tabular}{|c|c|c|c|c|c|c|c|c|c|}
\hline Q & \multicolumn{3}{|c|}{ Lena (512x512) } & \multicolumn{3}{c|}{ Barbara (512x512) } & \multicolumn{2}{c|}{ Peppers(512x512) } \\
\cline { 2 - 10 } JPEG & $\begin{array}{c}\text { Best } \\
\text { results } \\
\text { from } \\
{[\mathbf{1 3}]}\end{array}$ & $\begin{array}{c}\text { Propos } \\
\text {-ed }\end{array}$ & JPEG & $\begin{array}{c}\text { Best } \\
\text { results } \\
\text { from } \\
{[\mathbf{1 3}]}\end{array}$ & $\begin{array}{c}\text { Propo } \\
\text {-sed }\end{array}$ & JPEG & $\begin{array}{c}\text { Best } \\
\text { results } \\
\text { from } \\
{[\mathbf{1 3}]}\end{array}$ & $\begin{array}{c}\text { Propo } \\
\text {-sed }\end{array}$ \\
\hline 4 & 26.47 & 27.63 & $\mathbf{2 7 . 7 5}$ & 23.49 & 24.13 & $\mathbf{2 4 . 3 7}$ & 25.59 & 26.72 & $\mathbf{2 6 . 8 3}$ \\
\hline 6 & 28.25 & 29.22 & $\mathbf{2 9 . 4 9}$ & 24.49 & 25.08 & $\mathbf{2 5 . 1 9}$ & 27.32 & 28.22 & $\mathbf{2 8 . 3 4}$ \\
\hline 8 & 29.47 & 30.37 & $\mathbf{3 0 . 5 6}$ & 25.19 & 25.71 & $\mathbf{2 5 . 7 9}$ & 28.39 & 29.28 & $\mathbf{2 9 . 3 0}$ \\
\hline 10 & 30.41 & 31.17 & $\mathbf{3 1 . 3 8}$ & 25.79 & 26.27 & $\mathbf{2 6 . 3 1}$ & 29.17 & 29.94 & $\mathbf{2 9 . 9 8}$ \\
\hline 12 & 31.09 & 31.79 & $\mathbf{3 1 . 9 9}$ & 26.33 & $\mathbf{2 6 . 8 1}$ & $\mathbf{2 6 . 8 1}$ & 29.78 & 30.47 & $\mathbf{3 0 . 5 1}$ \\
\hline
\end{tabular}

In Table 1, the PSNR results of the proposed method is compared with the best PSNR results obtained by any of the methods $[5,13,23,24,25,26]$ as reported in [13]. The results show that for Lena, Barbara and Peppers images the proposed method outperforms the best results reported in [13].

In Table 2, the PSNR results of the proposed method is compared with the results of several well known decimated and non-decimated wavelet based image deblocking schemes [10,11,12,27]. The results show that the proposed scheme consistently outperforms all the methods given in Table 2. This suggests that the fitted curve in Fig. 1 may be considered for deblocking purpose for a wide range of quantization tables that include both JPEG and non-JPEG tables. Fig. 2 shows the visual results comparison between the proposed deblocking scheme and a well known method discussed in Ref. [12] which is based on overcomplete wavelet representation. The visual results show that our method can efficiently reduce the blocking artifacts while preserving true edges and textural information. 
The International Journal of Multimedia \& Its Applications (IJMA) Vol.4, No.1, February 2012

Table 2. PSNR comparison between $[10,11,12,27]$ and proposed method for restoration from block DCT Quantization for three different Quantization tables.

\begin{tabular}{|c|c|c|c|c|c|c|}
\hline \multirow{2}{*}{$\begin{array}{c}\mathbf{Q} \\
\text { Table }\end{array}$} & \multicolumn{6}{|c|}{ Lena $(512 \times 512)$} \\
\hline & Test Image & [11] & [27] & {$[10]$} & {$[12]$} & Proposed \\
\hline Q1 & 30.71 & 31.21 & 31.30 & 31.14 & 31.61 & 31.64 \\
\hline Q2 & 30.09 & 30.76 & 30.70 & 30.65 & 31.19 & 31.25 \\
\hline \multirow[t]{3}{*}{ Q3 } & 27.40 & 28.31 & 27.89 & 28.07 & 28.65 & 28.68 \\
\hline & \multicolumn{6}{|c|}{ Barbara $(512 \times 512)$} \\
\hline & Test Image & [11] & [27] & [10] & {$[12]$} & Proposed \\
\hline Q1 & 25.95 & 25.23 & 24.65 & 26.14 & 26.37 & 26.45 \\
\hline $\mathbf{Q 2}$ & 25.59 & 25.07 & 24.54 & 25.83 & 26.04 & 26.14 \\
\hline \multirow[t]{3}{*}{ Q3 } & 24.05 & 24.11 & 23.63 & 24.40 & 24.66 & 24.82 \\
\hline & \multicolumn{6}{|c|}{ Peppers(512x512) } \\
\hline & Test Image & [11] & [27] & {$[10]$} & {$[12]$} & Proposed \\
\hline Q1 & 30.40 & 30.65 & 31.15 & 30.99 & 31.33 & 31.42 \\
\hline Q2 & 29.82 & 30.23 & 30.56 & 30.52 & 30.97 & 30.98 \\
\hline Q3 & 27.22 & 28.19 & 27.86 & 28.14 & 28.55 & 28.58 \\
\hline
\end{tabular}

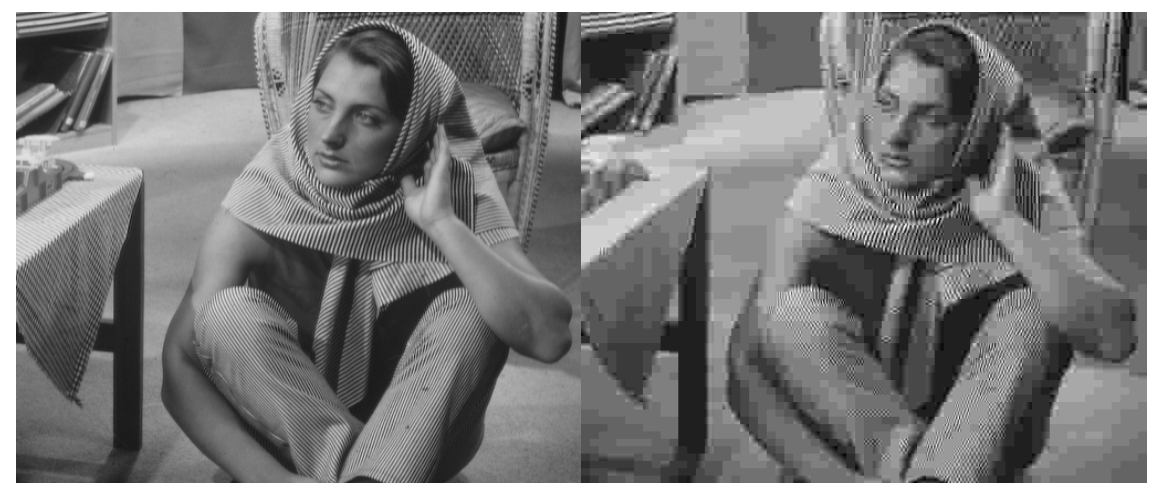

(a)

(b)

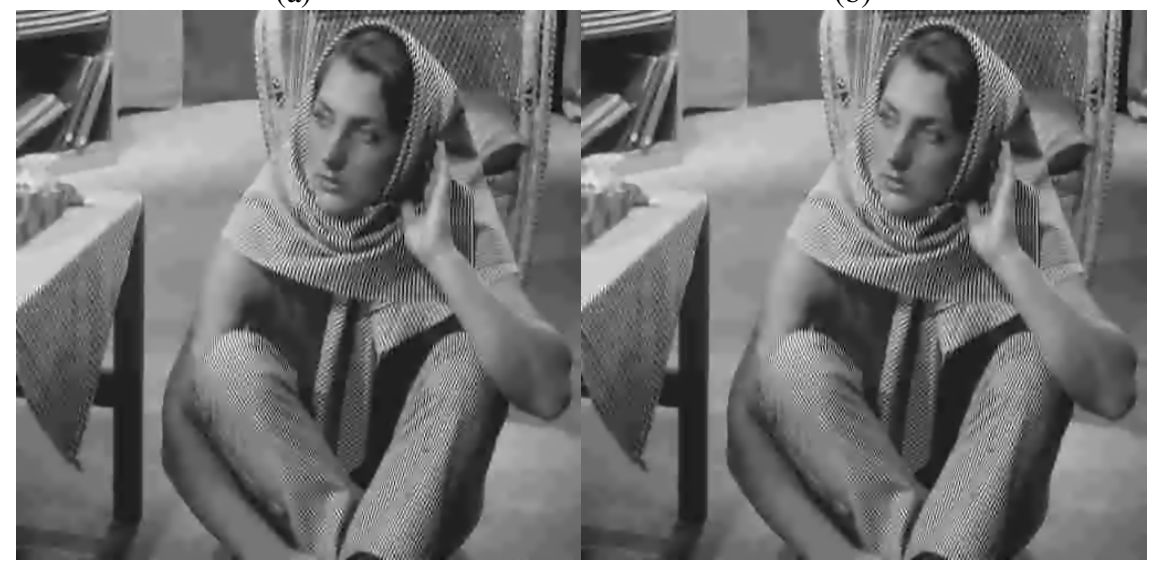

(c)

(d)

Figure 2. Deblocking results for Barbara image (a) Original (b) Block DCT compressed with Q2 $(\mathrm{PSNR}=25.59 \mathrm{~dB})(\mathrm{c})$ Processed with Liew and Yan's [12] algorithm $(\mathrm{PSNR}=26.04 \mathrm{~dB})(\mathrm{d})$ Processed with proposed method $(\mathrm{PSNR}=26.14 \mathrm{~dB})$ 
The International Journal of Multimedia \& Its Applications (IJMA) Vol.4, No.1, February 2012

\section{CONCLUSION}

We have proposed an efficient spatially adaptive wavelet transform domain image deblocking method which employs a MAP estimator that uses a Laplace pdf with local variance to reduce the blocking artifacts. The noise standard deviation is made adaptive to the quantization table used in the compression process and is calculated using an empirical expression. The local variance estimate of each coefficient is estimated from the local observation of the signal. The proposed method can suppress the blocking artifacts very efficiently while preserving the image details and object edges.

\section{REFERENCES}

[1] N. Ahmad, T. Natarajan, and K. Rao, "Discrete Cosine Transform," IEEE transactions on Computers, vol. C-23, no. 1, pp. 90-93, 1974.

[2] V. Bhaskaran and K. Konstantinides, Image and video compression standards - Algorithms and architectures, Kluwer Academic Publishers. 1997.

[3] H. Reeve and J. Lim, "Reduction of blocking effects in image coding," Opt. Eng., vol. 23, no. 1, pp. 34-37, 1984.

[4] S. Minami and A. Zakhor, "An optimization approach for removing blocking artifacts in transform coding," IEEE Transactions on Circuit and Systems for Video Technology. Vol. 5, pp. 74-82, 1995.

[5] Y. Yang, N. P. Galatsanos and A. K. Katsaggelos, “ Regularized reconstruction to reduce blocking artifacts of block discrete cosine transform compressed images", IEEE Transactions on Circuits and Systems for Video Technology. Vol.3, pp. 421-432, 1993.

[6] Y. Yang, N. P. Galatsanos, and A. K. Katsaggelos, "Projection based spatially adaptive reconstruction of block-transform compressed images,” IEEE Trans. Image Processing, vol. 4, no. 7, pp. 896-908, 1995.

[7] Y. Yang and N. P. Galatsanos, "Removal of compression artifacts using projections onto convex sets and line process modeling,” IEEE Transactions on Image Processing, Vol. 6, pp. 1345-1357, 1997.

[8] H. Paek, R. C. Kim and S. U. Lee, "On the POCS based postprocessing technique to reduce the blocking artifacts in transform coded images," IEEE Transactions on Image Processing, Vol. 8, pp. 358-367, 1998.

[9] J. Xu, S. Zheng and X. Yang, "Adaptive video blocking artifact removal in Discrete Hadamard Transform domain,” Optical Engineering, Vol. 45, 2006.

[10] S. Wu, H. Yan and Z. Tan, "An Efficient Wavelet-Based Deblocking Algorithm for Highly Compressed Images," IEEE Transactions on Circuits and Systems for Video Technology, vol.11, pp. 1193-1198, 2001.

[11] Z. Xiong, M. T. Orchard and Y. Zhang, "A deblocking algorithm for JPEG compressed images using overcomplete wavelet representation," IEEE Transactions on Circuits and Systems for Video Technology, vol. 77, pp. 433-437, 1997.

[12] A. W. C. Liew and H. Yan, "Blocking artifacts suppression in block coded images using overcomplete wavelet representation," IEEE Transactions on Circuits and Systems for Video Technology, vol.14, pp. 450-461, 2004.

[13] A. Z. Averbuch, A. Schclar and D. L. Donoho, "Deblocking of block transform compressed images using weighted sums of symmetrically aligned pixels," IEEE Transactions on Circuits and Systems for Video Technology, vol. 14, pp. 200-212, 2005. 
The International Journal of Multimedia \& Its Applications (IJMA) Vol.4, No.1, February 2012

[14] A. Foi, V. Katkovnik and K. Egiazarian, "Pointwise shape adaptive DCT for high quality denoising and deblocking of grayscale and color images," IEEE Transactions on Image Processing, vol.16,no.5, pp. 1395-1411, 2007.

[15] G. Zhai, W. Zhang, X. Yang, W. Lin and Y. Xu, "Efficient image deblocking based on postfiltering in shifted windows," IEEE Transactions on Circuits and Systems for Video Technology, vol.18, pp.122126,2008 .

[16] M. Zhang and G. K. Bahadir, "Compression Artifact Reduction with Adaptive Bilateral Filtering”, in Proc. Visual Communications and Image Processing. Vol. 7257, 2009.

[17] V. K. Nath, D. Hazarika and A. Mahanta, Blocking Artifacts Reduction Using Adaptive Bilateral Filtering, in Proc. International Conference on Signal Processing and Communications, vol.6, pp. 243- 250, 2010.

[18] V. K. Nath, A. Mahanta, “ Image denoising based on Laplace distribution with local parameters in Lapped transform domain", International Conference on Signal Processing and Multimedia Applications, Seville, Spain, July 2011.

[19] H. Rabbani and M. Vafadust, "Image / video denosing based on a mixture of Laplace distributions with local parameters in multidimensional complex wavelet domain," Signal Processing, vol. 88, no. 11, pp. 158-173, 2008.

[20] R. A. Gopinath, "Wavelet-based post-processing of low bit rate transform coded images," in IEEE International Conference on Image Processing, 1994, pp. 913-917.

[21] S. H. Park and D. S. Kim, "Theory of projection onto the narrow Quantization constraint set and its application,” IEEE Transactions on Image Processing, vol. 8, pp.1361-1373, 1999.

[22] I. J. Group. [Online]. Available: http://www.ijg.org.

[23] T. Chen, H. R. Wu and B. Qiu, "Adaptive postfiltering of transform coefficients for the reduction of blocking artifacts," IEEE Transactions on Circuits and Systems for Video Technology. vol. 11, pp.594-602, 2001.

[24] R. Castagno, S. Marsi and G. Ramponi, "A simple algorithm for the reduction of blocking artifacts in images and its implementation," IEEE Transactions on Consumer Electronics, vol.44, 1998.

[25] MPEG-4 video verification model version 18.0 (VM-18), ISO/IEC JTC1/SC29/WG11Std., 2001.

[26] R. Rosenholts and A. Zakhor, "Iterative procedures for reduction of blocking artifacts in transform domain image coding," IEEE Transactions on Circuits and Systems for Video Technology, vol. 2, pp. 91-95, 1992.

[27] T. C. Hsung and D. P. K. Lun, "Application of singularity detection for the deblocking of JPEG decoded images," IEEE Transactions on Circuits Systems II, vol.45, pp. 640-644, 1998. 